\title{
Papéis, conflitos e gratificações de enfermeiros especialistas em enfermagem psiquiátrica e saúde mental
}

\author{
Roles, conflicts and fulfillment of psychiatric and mental health nurse specialists
}

Papeles, conflictos y gratificaciones de enfermeros especialistas en enfermería psiquiátrica y salud mental

Raphael Valentino Marques de Lima ${ }^{1}$, Luiz Jorge Jorge Pedrão², Adriana Inocenti Miasso ${ }^{3}$, Moacyr Lobo da Costa Junior ${ }^{4}$

\section{RESUMO}

Este estudo descritivo-exploratório de cunho qualitativo teve por objetivo verificar quais os papéis, conflitos e gratificações de enfermeiros especialistas em enfermagem psiquiátrica e saúde mental, pois com as transformações da assistência psiquiátrica nas últimas décadas tornaram-se importantes essas compreensões. Assim, quinze enfermeiros, egressos de um curso de especialização em enfermagem psiquiátrica e saúde mental, responderam a um questionário com esse objetivo, e sua análise foi realizada de forma qualitativa. Os resultados mostraram que a maioria dos entrevistados é jovem, trabalha nessa área desempenhando papéis do enfermeiro e prestando cuidado direto. Constatou-se que esses enfermeiros possuem conflitos, como falta de reconhecimento, sobrecarga e falta de recursos humanos e gratificações, como a melhora do paciente e o reconhecimento profissional. Conclui-se, portanto, que os sujeitos identificam seu papel de especialista exercendo-o com propriedade; seus conflitos se relacionam a aspectos de reconhecimento e estrutura dos serviços; e suas gratificações estão ligadas a fatores de ordem pessoal e qualificação profissional. Descritores: Enfermagem Psiquiátrica; Saúde Mental; Desempenho de Papéis.

\section{ABSTRACT}

This descriptive-exploratory study was performed using a qualitative approach, with the objective to verify the roles, conflicts and fulfillment of psychiatric nursing and mental health nurse specialists, because these are relevant aspects considering the changes made in psychiatric care over the last decades. Therefore, fifteen nurses, graduates of a specialization course in psychiatric and mental health nursing, answered a questionnaire, which was subjected to qualitative analysis. The results showed that most subjects are young and work in the referred area, performing nursing roles and providing direct care. They reported experiencing conflicts, such as not being recognized, dealing with work overload and the lack of human resources. The aspects of fulfillment reported were the patients' improvement and professional recognition. Thus, it is concluded that the subjects identify their role as specialists and perform that role accordingly; their conflicts were related to aspects concerning recognition and the structure of the services, and their fulfillment is related to personal and professional qualification factors.

Descriptors: Psychiatric Nursing; Mental Health; Role Playing.

\section{RESUMEN}

Estudio descriptivo-exploratorio de cuño cualitativo que objetivó verificar cuáles son los papeles, conflictos y gratificaciones de enfermeros especialistas en enfermería psiquiátrica y salud mental, comprensiones importantes de acuerdo a las transformaciones de la atención psiquiátrica en las últimas décadas. Quince enfermeros egresados de curso de especialización en enfermería psiquiátrica y salud mental respondieron cuestionario con tal objetivo, el análisis realizado fue de carácter cualitativo. Los resultados mostraron que la mayoría de los entrevistados es joven, trabaja en ésta área desempeñando papeles de enfermero y brindando cuidado directo. Poseen conflictos, como falta de reconocimiento, sobrecarga y falta de recursos humanos y gratificaciones, tales como la mejoría del paciente y el reconocimiento profesional. Se concluye en que los sujetos identifican su papel de especialistas ejerciéndolo adecuadamente, sus conflictos se relacionan con aspectos de reconocimiento y estructura de servicios, y sus gratificaciones están vinculadas con factores de orden personal y calificación profesional.

Descriptores: Enfermería Psiquiátrica; Salud Mental; Desempeño de Papel.

\footnotetext{
1 Enfermeiro, Discente do Programa de Pós-Graduação em Enfermagem Psiquiátrica - nível Mestrado, Enfermeiro do CAPS III Novo Tempo da Prefeitura Municipal de Campinas. Campinas, SP, Brasil. E-mail: limarvm@usp.br.

2 Enfermeiro, Doutor em Enfermagem. Professor Doutor, Escola de Enfermagem de Ribeirão Preto (EERP), Universidade de São Paulo (USP). Ribeirão Preto, SP, Brasil. E-mail: lujope@eerp.usp.br.

${ }^{3}$ Enfermeira, Doutora em Enfermagem, Professora Doutora, EERP, USP. Ribeirão Preto, SP. E-mail: amiasso@eerp.usp.br.

${ }^{4}$ Estatístico, Doutor em Saúde Pública, Professor Associado, EERP, USP. Ribeirão Preto, SP. E-mail: mlobojr@eerp.usp.br.
} 


\section{INTRODUÇÃO}

Pode-se dizer que na enfermagem psiquiátrica a partir da década de 1960, especialistas como Ruth Virginia Matheney, Mary Topalis, Hildegard Peplau, Maria Aparecida Minzoni, Susan Irving, Joyce Travelbee, e, mais recentemente, no final do século $X X$ e início do século XXI, Cecelia Monat Taylor, Ruth Mylius Rocha, Gail Wiscarz Stuart e Michele Tereza Laraia, tem enfatizado, de alguma forma, que o papel do enfermeiro psiquiátrico é o de agente terapêutico e suas ações têm base no relacionamento estabelecido com a pessoa com diagnóstico de transtorno mental que assiste e é entendido como relação terapêutica(1-2).

Salienta-se que isto vem sendo preconizado como um modelo no ensino de enfermagem psiquiátrica, mas, o enfermeiro que se encontra na prática, vem tendo dificuldades para desenvolvê-lo, indicando que a relação terapêutica, ou foi delegada a outros da equipe de enfermagem, ou não está sendo realizada pelos enfermeiros, que se ocupam em diversos outros papéis, como os administrativos, por exemplo(3-6).

Percebe-se então, certa dicotomia entre o que se propõe teoricamente e o que se realiza na prática. Mesmo o enfermeiro realizando tarefas administrativas, era de se esperar que esta atividade estivesse relacionada à administração da assistência ou do cuidado direto que fora delegado, preservando-se assim o caráter transformador destes profissionais.

Esses aspectos no geral podem levar o enfermeiro, em particular o enfermeiro psiquiátrico, a ter alguns conflitos ou insatisfações no desempenho de suas funções, principalmente pelo fato de que a escolha pela profissão de enfermagem não tem base íntegra nas razões vocacionais. O nível salarial, a qualidade da supervisão, o relacionamento com a equipe de trabalho, as condições de trabalho propriamente e as atitudes e comportamentos congruentes com o papel, são também apontados como fatores de insatisfação, entre outros $^{(4,7-8)}$. Por outro lado, o reconhecimento, a responsabilidade e a autonomia são apontados pelo enfermeiro como fatores que oferecem algum estado de satisfação ${ }^{(7-9)}$.

Nas últimas décadas, a transformação da psiquiatria vem ocorrendo, trazendo consigo exigências no sentido de que os seus profissionais busquem, além de melhor formação, também uma atualização no sentido de acompanhar todo o processo de mudança, fazendo parte deste contexto a enfermagem psiquiátrica e os seus profissionais. As reais mudanças começaram a partir da década de 60 , onde as ações de enfermagem começaram a ter base no relacionamento terapêutico influenciado tanto pela Psiquiatria Social, que faz críticas ao modelo asilar e propõe a sua transformação no sentido de tornar-se um ambiente onde a saúde mental pudesse emergir, quanto pela Comunidade Terapêutica, que considera fundamental o treinamento da equipe, o tipo de relacionamento que se estabelece na comunidade e o papel ativo e responsável que o paciente é estimulado a assumir ${ }^{(10-12)}$.

Assim sendo, o enfermeiro deve exercer as suas funções profissionais vinculadas aos programas construídos dentro deste processo de transformação, o que implica em criar novos espaços para assistência de enfermagem psiquiátrica e de saúde mental no sentido de acolher o portador de transtornos mentais fora do espaço antes restrito, que se traduzia nos grandes hospitais psiquiátricos ${ }^{(10-12)}$.

As novas alternativas de assistência aos portadores de transtornos mentais, após as propostas da diminuição do número de leitos nos grandes hospitais psiquiátricos e o seu fechamento gradativo, estimulando à assistência fora deste contexto de serviço fechado e buscando uma assistência integral em serviços abertos, levou também o enfermeiro a se adequar a esse novo contexto.

Considerando esse panorama, desenvolveu-se o presente estudo, cujo objetivo foi identificar os papéis, conflitos e gratificações de enfermeiros especialistas em enfermagem psiquiátrica e saúde mental.

\section{MÉTODO}

O presente estudo é de natureza descritivo exploratório com análise qualitativa dos dados, o que permite o estudo das relações, representações, crenças, percepções e opiniões, produtos das interpretações que os indivíduos fazem do seu modo de viver, sentir e pensar. Esta abordagem é entendida como mais adequada aos estudos de grupos, onde se pretende captar a visão dos sujeitos frente aos acontecimentos sociais $^{(13)}$.

O projeto de pesquisa no qual o presente estudo está inserido, foi apreciado e aprovado pelo Comitê de Ética em Pesquisa Envolvendo Seres Humanos do Hospital das Clínicas da Faculdade de Medicina de 
Ribeirão Preto da Universidade de São Paulo (HCFMRPUSP) - Processo HCRP n³ 3871/2006.

Constituíram-se como participantes todos os enfermeiros de uma turma que concluiu um Curso de Especialização em Enfermagem Psiquiátrica e Saúde Mental tradicional, no ano de 2006. A pesquisa foi realizada no ano de 2009 , onde, primeiramente, na secretaria do referido curso, foi feita uma busca em seus arquivos para levantamento de todos os enfermeiros que o cursaram no ano proposto para a realização da pesquisa. Assim, nessa turma, havia um número de 20 enfermeiros matriculados, e, destes, 19 concluíram o curso, sendo assim, considerados os enfermeiros egressos. Posteriormente, os enfermeiros especialistas identificados, foram rastreados, e, após sua localização, foi feito um convite para a participação através de carta, e-mail ou telefone.

Para os que aceitaram participar, foi enviado pelo correio o material para coleta dos dados, que incluiu: Termo de Consentimento Livre e Esclarecido; Questionário para coleta dos dados da pesquisa, com duas partes, sendo a primeira com questões sobre os dados pessoais, e a segunda, com as seguintes questões: enquanto enfermeiro especialista em enfermagem psiquiátrica e saúde mental, quais os papéis que você desenvolve? Quais os conflitos que você tem no trabalho que desenvolve enquanto enfermeiro especialista em enfermagem psiquiátrica e saúde mental? Quais as gratificações que você tem no trabalho que desenvolve enquanto enfermeiro especialista em enfermagem psiquiátrica e saúde mental?

Juntamente com todo o material, foi encaminhado também um envelope selado para a devolução do questionário, já endereçado aos pesquisadores.

A análise do material proveniente das respostas dos questionários foi feita, primeiramente, a partir de leituras exaustivas, e, sequencialmente, realizadas sínteses para as respostas de cada uma das questões. Posteriormente, o material sintetizado foi agrupado por semelhança de seus conteúdos, o que permitiu o estabelecimento de categorias definidoras, que foram apresentadas na forma de tabelas, a fim de facilitar a visualização(13).

Utilizou-se a escrita como código linguístico, mais especificamente a comunicação dialógica entre os pesquisadores e os sujeitos, por meio do questionário semi-estruturado, e, assim, foi feita uma análise de seus significados, tendo como preceito da análise temática. A análise de conteúdo aparece como um conjunto de técnicas de análise das comunicações que utiliza procedimentos sistemáticos e objetivos de descrição do conteúdo das mensagens, buscando conhecer aquilo que está por trás das palavras as quais se debruça(14).

\section{RESULTADOS}

Os resultados serão apresentados na forma de tabelas, conforme já referido, buscando uma facilitação no sentido de melhor visualização dos mesmos. Achouse interessante apresentar o número de respostas dadas em cada categoria, no sentido de mostrar o número de enfermeiros que responderam em cada uma delas, mas, não foi intenção neste estudo, quantificar essas respostas, o que poderia caracterizar um estudo também quantitativo. Assim, os dados numéricos apresentados têm apenas a finalidade de complemento, sendo o estudo de natureza descritivo exploratório, com análise qualitativa de seus resultados.

\section{Caracterização dos participantes do estudo}

De um total de 19 envelopes enviados contendo o material para os possíveis participantes, 15 profissionais, todos do sexo feminino, retornaram os questionários e assinaram o termo de Consentimento Livre e Esclarecido, e, destes que aceitaram participar, 13 foram analisados integralmente. Os outros dois questionários não apresentaram respostas, assim, foi possível apenas a utilização de seus dados pessoais. A Tabela 1 apresentada a seguir, mostra os participantes da pesquisa e seu perfil profissiográfico. 
Tabela 1: Distribuição dos especialistas participantes por idade, tempo de formação, tempo de trabalho e capacitação profissional ( $n=15)$. Ribeirão Preto, SP, 2009.

\begin{tabular}{|c|c|c|c|c|c|c|}
\hline \multirow{2}{*}{ Enfermeiro (E) } & \multirow{2}{*}{$\begin{array}{l}\text { Idade em } \\
\text { anos }\end{array}$} & \multirow{2}{*}{$\begin{array}{l}\text { Tempo de } \\
\text { formado em } \\
\text { anos }\end{array}$} & \multirow{2}{*}{$\begin{array}{l}\text { Tempo que } \\
\text { exerce a } \\
\text { profissão em } \\
\text { anos }\end{array}$} & \multirow{2}{*}{$\begin{array}{l}\text { Trabalha na área de } \\
\text { enfermagem } \\
\text { psiquiátrica e ou } \\
\text { saúde mental? }\end{array}$} & \multicolumn{2}{|c|}{$\begin{array}{c}\text { Pós - Graduação } \\
\text { Stricto Sensu }\end{array}$} \\
\hline & & & & & Mestrado & Doutorado \\
\hline 1 & 28 & 3 & 1 & Sim & Sim & Não \\
\hline 2 & 34 & 11 & 11 & Sim & Não & Não \\
\hline 3 & 28 & 5 & 4 & Não & Não & Não \\
\hline 4 & 28 & 3 & 2 & Não & Não & Não \\
\hline 5 & 32 & 3 & 3 & Sim & Não & Não \\
\hline 6 & 32 & 3 & 2 & Não & Não & Não \\
\hline 7 & 26 & 2 & 1 & Sim & Sim & Não \\
\hline 8 & 24 & 3 & 3 & Não & Não & Não \\
\hline 9 & 36 & 4 & 2 & Sim & Não & Não \\
\hline 10 & 53 & 4 & 2 & Sim & Não & Não \\
\hline 11 & 24 & 2 & 2 & Não & Não & Não \\
\hline 12 & 40 & 3 & $<1$ & Não & Não & Não \\
\hline 13 & 33 & 8 & 8 & Sim & Não & Não \\
\hline 14 & 29 & 3 & 3 & Sim & Não & Não \\
\hline 15 & 27 & 3 & 3 & Sim & Não & Não \\
\hline
\end{tabular}

Conforme mostra a Tabela 1, a grande maioria dos profissionais sujeitos do estudo está na faixa etária entre 21 e 40 anos, constituindo-se em 93,3\% do total de participantes, e, a maioria absoluta dos enfermeiros pesquisados (60\%) declarou desempenhar suas atividades profissionais na área de enfermagem psiquiátrica e saúde mental. O tempo de formado variou entre três e 11 anos e o tempo que exercem a profissão variou entre menos de um ano e 11 anos. Dois enfermeiros especialistas em enfermagem psiquiátrica e saúde mental possuem curso de Pós-Graduação Stricto Sensu nível Mestrado, e nenhum possui nível Doutorado.

\section{Papéis}

Os conteúdos referentes aos papéis foram agrupados em quatro categorias definidoras, sendo elas: administrativo, mencionada por três enfermeiros; papéis de cuidado direto, descrito por 10 enfermeiros; papéis específicos, referida por 11 enfermeiros e docência, citada por dois enfermeiros, conforme mostra a Tabela 2.

Tabela 2: Distribuição das situações conforme as categorias definidoras dos papéis. Ribeirão Preto, SP, 2009.

\begin{tabular}{|c|c|c|}
\hline Papéis/Categorias & Situações Relacionadas aos Papéis & $\begin{array}{l}\text { Número de } \\
\text { Respostas }\end{array}$ \\
\hline Administrativo & $\begin{array}{l}\text { Coordenação dos serviços de assistência; organização do serviço de assistência; reuniões; } \\
\text { educação continuada; organização de eventos; confecção de manuais. }\end{array}$ & 3 \\
\hline $\begin{array}{c}\text { Papéis de cuidado } \\
\text { direto }\end{array}$ & $\begin{array}{l}\text { Atuar junto ao paciente e aos familiares; execução dos cuidados (banho, medicações e } \\
\text { orientações); apoio psicológico ao paciente; controle de crises; acompanhamento dos } \\
\text { familiares; acompanhamento dos pacientes; assistência aos pacientes. }\end{array}$ & 10 \\
\hline & $\begin{array}{l}\text { Elaboração do processo de enfermagem; desenvolvimento da escala mensal e diária dos } \\
\text { funcionários; liderança da equipe preservando a integração e harmonia; } \\
\text { coordenação/supervisão/orientação da equipe de enfermagem; consulta de } \\
\text { enfermagem; pós-consulta de enfermagem; aplicação do exame do estado mental; }\end{array}$ & \\
\hline Papéis específicos & $\begin{array}{l}\text { triagem; acolhimento; visita domiciliar; prevenção na comunidade; contra-referência; } \\
\text { escuta terapêutica; defesa do cliente e da família; controle da medicação; } \\
\text { desenvolvimento de rotinas de atividades; encaminhamento para outra especialidade; } \\
\text { membro da equipe multiprofissional; profissional de referência; desenvolvimento e } \\
\text { participação de grupos temáticos e oficinas. }\end{array}$ & 11 \\
\hline Docência & $\begin{array}{c}\text { Administração de aulas para cursos profissionalizantes de técnicos e auxiliares de } \\
\text { enfermagem; professor supervisor para graduandos. }\end{array}$ & 2 \\
\hline
\end{tabular}

Obs.: um mesmo participante respondeu mais de um papel.

Na categoria papel administrativo, a atividade a ela relacionada apareceu nas respostas de três dos participantes, e, as atividades relativas à categoria dos papéis de cuidado direto, foram descritas por 10 dos enfermeiros especialistas participantes. 
Na categoria papéis específicos, as atividades foram descritas por 11 enfermeiros, sendo que, a docência, foi uma categoria criada para agrupar as atividades de ensino e mencionadas por apenas dois enfermeiros psiquiátricos participantes.

\section{Conflitos}

Os conteúdos referentes aos conflitos, foram agrupados em cinco categorias, sendo elas: o reconhecimento profissional, onde todas as respostas foram dadas por quatro dos enfermeiros especialistas participantes; o conflito nas condições de trabalho foi descrito por três dos sujeitos da pesquisa; categoria relacionamento com a equipe foi descrita como conflito por três dos profissionais participantes; recursos humanos desqualificados e falta de capacitação profissional, apontado por seis especialistas, revelou-se como a categoria de conflitos que teve o maior número de citações desta categoria definidora, e, uma categoria interessante, foi a de profissionais que não tem conflitos no serviço, referido por dois dos enfermeiros participantes. A Tabela 3 apresentada a seguir, mostra esses resultados.

Tabela 3: Distribuição das situações conforme as categorias definidoras dos conflitos. Ribeirão Preto, SP, 2009.

\begin{tabular}{|c|c|c|}
\hline Conflitos/Categorias & Situações Relacionadas aos Conflitos & $\begin{array}{l}\text { Número de } \\
\text { Respostas }\end{array}$ \\
\hline Reconhecimento Profissional & $\begin{array}{l}\text { falta de reconhecimento como enfermeiro especialista; não diferenciação } \\
\text { entre enfermeiros generalistas e especialistas; não credibilidade nas } \\
\text { decisões; falta de credibilidade no atendimento do enfermeiro } \\
\text { especialista; falta de reconhecimento financeiro. }\end{array}$ & 4 \\
\hline Condições de Trabalho & $\begin{array}{l}\text { falta de recursos materiais e de espaço físico adequado; falta de recursos } \\
\text { humanos; sobrecarga do profissional qualificado. }\end{array}$ & 3 \\
\hline Relacionamento com a equipe & $\begin{array}{l}\text { profissionais médicos que não conseguem trabalhar em equipe; falta de } \\
\text { cooperação entre enfermeiros. }\end{array}$ & 3 \\
\hline $\begin{array}{l}\text { Recursos humanos } \\
\text { desqualificados e falta de } \\
\text { capacitação profissional }\end{array}$ & $\begin{array}{l}\text { falta de qualificação dos profissionais em geral; falta de capacitação da } \\
\text { equipe; falta de tato tanto da equipe medica como da equipe de } \\
\text { enfermagem; grande rotatividade dos profissionais no serviço. }\end{array}$ & 6 \\
\hline Não tem conflitos & não teve conflito até hoje no serviço. & 2 \\
\hline
\end{tabular}

\section{Gratificações}

Os conteúdos relativos às gratificações foram agrupados em quatro categorias, sendo elas: reconhecimento profissional, cujas situações foram levantadas por nove enfermeiros especialistas; o reconhecimento pessoal, com situações mencionadas por sete participantes; qualificação, que agrupou situações referidas por três dos sujeitos inclusos no estudo, e a categoria relacionamento, que foi lembrada por apenas um enfermeiro especialista participante, conforme a Tabela 4 apresentada a seguir.

Tabela 4: Distribuição das situações conforme as categorias definidoras das gratificações. Ribeirão Preto, SP, 2009.

\begin{tabular}{lcc}
\hline Gratificações/Categorias & Situações Relacionadas às Gratificações & Número de \\
Respostas
\end{tabular}

\begin{tabular}{cl}
\hline $\begin{array}{c}\text { Reconhecimento } \\
\text { profissional }\end{array}$ & $\begin{array}{l}\text { agradecimento do paciente pelos serviços prestados; melhora do paciente; } \\
\text { reconhecimento da equipe multiprofissional pelo meu trabalho; } \\
\text { reconhecimento dos pacientes; promoção de cargo; aumento do mercado de } \\
\text { trabalho para profissionais qualificados. }\end{array}$ \\
satisfação pessoal; ser reconhecido como enfermeiro psiquiátrico; \\
gratificaçães de alunos e colegas; diferenciação no gerenciamento de \\
pessoal; prazer em trabalhar; descoberta da aptidão; bom desempenho do \\
meu papel. \\
aplicação dos conhecimentos; segurança no atendimento; postura; ética; \\
interesse em buscar mais qualificação; olhar diferenciado sobre o cuidado em \\
saúde mental; qualificação para trabalhar em serviços extra hospitalares.
\end{tabular}

Obs.: um mesmo participante respondeu mais de uma gratificação. 


\section{DISCUSSÃo}

A escolha de um tradicional curso de especialização em enfermagem psiquiátrica e saúde mental para identificar os papéis, conflitos e gratificações dos enfermeiros dele egressos foi oportuno, pois, assim, pode-se assegurar a idoneidade da formação(15). No transcorrer desta discussão, alguns trechos de falas dos enfermeiros participantes da presente pesquisa serão utilizados, pelo entendimento de que esse procedimento se constitui em uma forma ilustrativa de salientar as passagens que estão sendo discutidas.

Estudar apenas uma turma constituiu-se em um limite do estudo, mas possibilitou ter-se uma noção do envolvimento dos enfermeiros especializados na área em questão com a própria área, pois, através de aspectos presentes no cotidiano dos profissionais estudados, como a identificação de seus papéis, conflitos e gratificações relacionados ao desenvolvimento de suas funções, foi possível a verificação deste envolvimento, ficando isto evidente, pois a maioria dos enfermeiros pesquisados atua na área em que se especializaram. Os que não são envolvidos com a área citada são os mais novos e com tempo menor de exercício da profissão, o que pode levar ao entendimento de que ainda estão em busca de oportunidades para atuar nesta área. Pesquisar outras turmas e de forma comparativa, parece ser interessante e constitui-se em uma sugestão.

Importante destacar que entre os 15 enfermeiros especialistas estudados dois têm curso de PósGraduação Stricto Sensu nível Mestrado, o que se constitui em um bom número, levando em consideração que estão vinculados a serviços de assistência e não ao ensino ou pesquisa, onde este tipo de formação se faz mais necessária.

No contexto atual da atuação do enfermeiro nas equipes multiprofissionais dos diversos serviços de assistência psiquiátrica, a afirmação de que o trabalho efetivo dos enfermeiros centra-se, principalmente, no desenvolvimento de atividades burocráticoadministrativas $^{(16)}$, é verdadeira. Mas, os resultados do presente estudo mostraram que, os enfermeiros participantes, possuem uma postura diferente, que não vai ao encontro desta afirmação, aproximando-se mais do princípio de que o papel do enfermeiro é realmente o que se encontra na proposta de atuação preconizada pela reabilitação psicossocial, onde é dito que os portadores de diagnóstico de transtorno mental tem direito a atendimento individual (medicamentoso, psicoterápico, de orientação, entre outros), atendimento em grupos (psicoterapia, grupo operativo, atividades de suporte social, entre outras), atendimento em oficinas terapêuticas executadas por profissional de nível superior ou nível médio, visitas domiciliares, atendimento à família, atividades comunitárias enfocando a integração do paciente na comunidade e sua inserção familiar e social(17). Assim sendo, as respostas apontam para uma atuação dos enfermeiros mais voltada aos cuidados diretos e específicos do que aos burocráticos(7) e, isto, fica evidente na resposta de um dos sujeitos do estudo, citada a seguir.

Tenho função de liderar a equipe, planejar, implementar $e$ avaliar a assistência de enfermagem de cada paciente, preservar a harmonia e integração da equipe e fazer com que ela tenha em mente que são responsáveis pela assistência e bem estar do paciente e o principal atuar junto ao paciente e a família, atendendo suas necessidades básicas.(E4)

A fala deste enfermeiro sobre sua percepção de papel vem em consonância com o que é preconizado pelo Ministério da Saúde para a reabilitação psicossocial sobre a assistência aos portadores de transtornos mentais, onde refere o direito ao atendimento individual, atendimento em grupos, atendimento em oficinas terapêuticas executadas por profissional de nível superior ou nível médio, visitas domiciliares, atendimento à família, atividades comunitárias enfocando a integração do paciente na comunidade e sua inserção familiar e social(17). Um dos enfermeiros fez a seguinte colocação quando respondeu sobre qual o papel desenvolvia:

Trabalho em um CAPS III e desenvolvo em parceria com mais um profissional três oficinas..., além das atividades de núcleo de enfermagem como organização do serviço $e$ outras atividades.(E15)

Esta resposta mostra que o desenvolvimento de oficinas é reconhecido pelos enfermeiros como um dos papéis de sua competência em serviços de atenção a saúde mental e que pode ser compartilhado, muito comum em serviços abertos de assistência psiquiátrica como os Centros de Atenção Psicossociais. O enfermeiro, 
inserido nas equipes multiprofissionais dos serviços de saúde mental, participa das atividades definidas por esta equipe, interfere e conduz o processo de atendimento e seguimento dos portadores de transtornos mentais, como qualquer outro profissional da referida equipe e orienta, ainda, a equipe de enfermagem, atendendo às especificidades da profissão. Vive, assim, a experiência de um trabalho inovador, integrado à equipe do serviço de saúde mental onde está inserido, contribuindo, como qualquer outro profissional desta equipe, para a melhoria do atendimento ${ }^{(1,18)}$.

A pesquisa mostra que oito enfermeiros dos 15 que participaram têm entre 21 e 30 anos de idade, com uma média de idade de 26.7 anos. Além disso, estão nessa faixa etária os únicos dois profissionais que possuem pós-graduação Stricto Sensu nível Mestrado, o que sugere que cada vez mais cedo esses profissionais estão buscando maior qualificação. Tal fato pode vir para responder às necessidades do seu local de trabalho, ou para a busca de melhor qualificação visando a ascensão profissional. Vale salientar que 93,3 dos enfermeiros pesquisados estão na faixa etária entre 21 e 40 anos, ou seja, uma faixa etária onde os profissionais reúnem condições excepcionais de produção, tanto no sentido de execução de técnicas e procedimentos como na avaliação dessas técnicas e procedimentos no sentido de aperfeiçoamento e, ainda, criação de novas técnicas, procedimentos e teorias, pois o curso de especialização contribui para esses aspectos e, no grupo pesquisado, haviam dois participantes com nível Mestrado.

Assim, os recursos humanos desqualificados e a falta de capacitação dos profissionais que desempenham suas atividades em conjunto com os entrevistados, foi apontado como um grande conflito, conforme colocações que seguem.

...quando ocorrem internações de pacientes psiquiátricos ou depressivos por problemas clínicos, percebo certa intolerância e falta de tato tanto da equipe de enfermagem quanto da equipe médica...(E8)

...interferência política que empregam funcionários sem formação em saúde mental,... Ausência de formação dos demais profissionais..., rotatividade de profissionais de nivel superior...(E13)

...de um modo geral é a limitada gama de recursos humanos de boa qualidade.... (E6)
Nesse sentido o que se pode imaginar é que, pela falta de qualificação dos profissionais, os serviços em geral estão deixando de dar o atendimento integral e de qualidade aos portadores de transtornos mentais ou aqueles que se encontram em estado de sofrimento psíquico. Esse grande número de profissionais desqualificados empregados pode se dar pelo fato de ser seu primeiro emprego ou pela falta de oportunidade de emprego em outras áreas.

Os enfermeiros entrevistados sentem-se fortemente gratificados com o reconhecimento profissional, pois apareceram em muitas das respostas que o que mais é gratificante para eles é ser reconhecido por parte da equipe e por parte do portador de transtorno mental, como um profissional qualificado e competente, evidenciado nas respostas apresentadas abaixo.

...reconhecimento dos outros profissionais pelo meu trabalho diferenciado, reconhecimento dos próprios pacientes/usuários...(E7)

...As gratificações como enfermeira psiquiátrica é ser reconhecida como tal e respeitada por isso...(E8)

As gratificações não são monetárias e sim um [muito obrigado, me sinto melhor agora, ou obrigada por me ouvir, você me ajudou muito] vindo dos pacientes...(E1)

Essas respostas podem carregar certo grau de idealismo, pois, além de respostas como essas, alguns sujeitos responderam, de forma entusiasmada, quando lhes foi perguntado quais eram as gratificações que tinham no trabalho, e a resposta foi a seguinte:

Todas, pois amo o que faço, amo a psiquiatria.(E2)

O fato de que esses enfermeiros têm sua maior gratificação no seu reconhecimento pessoal, deve se dar ao fato de que, por serem especialistas, estão mais qualificados, tem mais tempo de estudo na área e talvez por isso vejam esse reconhecimento profissional como o reconhecimento do seu tempo de estudo dedicado a esta área específica.

Por fim, destaca-se que o papel do enfermeiro psiquiátrico tem crescido nos últimos anos e vem sendo cada vez mais reconhecido pelos outros profissionais, principalmente em hospitais gerais, desenvolvendo interconsulta de enfermagem psiquiátrica e prestando apoio a outras áreas, como na clínica médica e 
cirúrgica ${ }^{(19)}$ e, ainda, em serviços abertos de assistência psiquiátrica, de um modo geral(20). Pelas respostas, os conflitos ainda merecem uma reflexão maior, pois era de se esperar que houvessem mais, devido principalmente a realidade da assistência à saúde mental no Brasil de um modo geral, apesar de grandes esforços dos serviços e profissionais de um modo geral, e, as respostas para as gratificações, mostraram que os enfermeiros psiquiátricos pesquisados respondem em um tom carregado de sentimento voltado principalmente à parte afetiva, o que se constitui em uma grande virtude.

\section{CONSIDERAÇÕES FINAIS}

Os resultados do presente estudo mostraram que o enfermeiro tem reconhecimento do seu papel na prática profissional, tanto os que estão trabalhando em serviços de assistência psiquiátrica, quanto os que desempenham suas atividades em outros serviços, resgatando e colocando em prática os conhecimentos adquiridos na sua especialização, o que faz deste enfermeiro um profissional de referência em suas ações profissionais. Não estão restritos em papéis burocráticos e administrativos, se colocam como agentes do cuidado direto, com papéis reais de assistência direta por inúmeras vezes, possibilitando a conclusão de que os enfermeiros estão conscientes de suas funções e de suas competências, o que demonstra um caráter de propriedade, maturidade e firmeza em suas ações dentro do ambiente de trabalho, particularmente voltados aos princípios da reabilitação psicossocial, relacionamento interpessoal, e amparo aos familiares e pacientes que estejam passando por sofrimento psíquico intenso independente do foco de ação das instituições onde estão vinculados.

O idealismo pode explicar o fato de que suas gratificações se resumem bastante em reconhecimento profissional/pessoal e não a seu reconhecimento financeiro, o que é particularmente importante, pois volta à atenção desse profissional ao objetivo da assistência e ao cuidado, deixando em segundo plano a valorização profissional pela remuneração, mesmo depois de sua pós-graduação, curso este que pode ter sido uma forma de agregar valor monetário ao currículo deste profissional, por sua melhor qualificação. Destacase que, mesmo em segundo plano, essas questões não devem ser esquecidas, pois a enfermagem continua sendo uma profissão muito desvalorizada em termos de remuneração, frente ao seu grande valor no cuidado à saúde de um modo geral, independente da modalidade de assistência, apesar do reconhecido crescimento das necessidades e atribuições profissionais.

Considera-se, finalmente, que o enfermeiro sabe qual o seu papel de especialista e o desempenha com propriedade, que os seus maiores conflitos são os recursos humanos desqualificados e a falta de capacitação profissional, que na sua ótica deterioram o atendimento aos portadores de transtornos mentais, tanto de serviços especializados em psiquiatria e saúde mental, como usuários de outros serviços, como ambulatoriais, clínicos ou serviços de emergência. Suas gratificações mais importantes estão relacionadas ao reconhecimento do seu trabalho enquanto enfermeiro especialista, tanto o reconhecimento pela sua própria equipe de enfermagem, da equipe multidisciplinar e o reconhecimento dos pacientes e usuários dos serviços. De um modo geral, são reconhecimentos sobre os seus serviços prestados, manifestados na forma de agradecimento verbal pela evolução positiva do quadro do usuário, fazendo destaque à sua atuação tanto pelo seu cuidado direto, quanto pela sua presença na organização do serviço, e, ainda, na orientação de sua equipe.

Como limites do presente estudo, pode-se considerar que apenas uma turma foi estudada, sendo importante a sua ampliação com dados de outras turmas. A análise qualitativa se mostrou adequada, mas associar dados quantitativos certamente pode conferir mais precisão aos resultados, inclusive de forma comparativa, permitindo uma discussão mais aprofundada.

3. Kirschbaum DIR, Paula FKC. O trabalho do enfermeiro nos equipamentos de saúde mental da rede pública de Campinas-SP. Rev. Latino-Am Enfermagem. 2001;9(5):77-82.

4. Diaz Heredia LP, Marziale MHP. El papel de los profesionales en centros de atención en drogas en ambulatorios de la ciudad de Bogotá, Colombia. Rev. Latino-Am Enfermagem. 2010;18(spec):57381. 
5. Filizola CLA. O papel do enfermeiro psiquiatra - oprimido e opressor. Rev. Esc. Enferm. USP. 1997;31(2):173-90.

6. Rosenstock KIV, Neves MJ. Papel do enfermeiro da atenção básica de saúde na abordagem ao dependente de drogas em João Pessoa, PB. Rev. Bras. Enfermagem. 2010;63(4):581-6.

7. Seed MS, Torkelson DJ, Alnatour R. The role of the inpatient psychiatric nurse and its effect on job satisfaction. Issues Ment Health Nurs. [Internet]. 2010 [cited 2011 set 20]; 31(3):160-70. Available from:

http://informahealthcare.com/doi/full/10.3109/0161284090316872 $\underline{9}$.

8. Lunardi Filho WD. Prazer e sofrimento no trabalho: contribuições à organização do processo de trabalho da enfermagem. Rev. Bras. Enfermagem. 1997;50(1):77-92.

9. Cura MLAD, Rodrigues ARF. Satisfação profissional do enfermeiro. Rev. Latino-Am Enfermagem. 1999;7(4):21-8.

10. Peres MAA, Barreira IA. Uma nova enfermagem psiquiátrica na universidade do Brasil nos anos 60 do século XX. Esc. Anna Nery Rev. Enferm. 2008;12(1):108-14.

11. Fernandes JD, et al. Ensino da enfermagem psiquiátrica/saúde mental: sua interface com a Reforma Psiquiátrica e diretrizes curriculares nacionais. Rev. Esc. Enferm. USP. 2009;43(4):962-8. 12. Munari DB, Godoy MTH, Esperidião E. Ensino de enfermagem psiquiátrica / saúde mental na Faculdade de Enfermagem da Universidade Federal de Goiás. Esc. Anna Nery Rev. Enferm. 2006;10(4):684-93.

13. Minayo MCS. O desafio do conhecimento: pesquisa qualitativa em saúde. $12^{\mathrm{a}}$ ed. São Paulo (SP): Hucitec, 2010.

14. Bardin L. Análise de Conteúdo. Lisboa: Edições 70, 1977.

15. Furegato ARF, Saeki T. Formando especialistas em enfermagem psiquiátrica e saúde mental por mais de duas décadas. In: Cadernos do IPUB. Rio de Janeiro 2000; VI(17): 128.41.

16. Oliveira AGB, Alessi NP. O trabalho de enfermagem em saúde mental: contradições e potencialidades atuais. Rev. Latino-Am Enfermagem. 2003;11(3):333-40.

17. Ministério da Saúde, (BR). Portaria n. 336/GM de 19 de fevereiro de 2002. Estabelece que os Centros de Atenção Psicossocial poderão constituir-se nas seguintes modalidade de serviços: CAPS I, CAPS II e CAPS III, definidos por ordem crescente de porte/complexidade e abrangência populacional. Brasília: Ministério da Saúde, 2002.

18. Silveira MR, Alves M. O enfermeiro na equipe de saúde mental - o caso dos CERSAMS de Belo Horizonte. Rev. Latino-Am Enfermagem. 2003;11(5):645-51.

19. Scherer ZAP, Scherer EA, Labate RC. Interconsulta em enfermagem psiquiátrica: qual a compreensão do enfermeiro sobre esta atividade? Rev. Latino-Am Enfermagem. 2002;10(1):7-14.

20. Lima RVM, Pedrão LJ, Gonçalves JG, Luis MAV. Papéis, conflitos e gratificações do enfermeiro de serviços abertos de assistência psiquiátrica. Rev. Eletr. Enf. [Internet]. 2010 [cited 2011 jun 15];12(2);348-53. Available from: http://www.fen.ufg.br/revista/v12/n2/v12n2a19.htm.

Artigo recebido em 15.10.2010.

Aprovado para publicação em 13.03.2012.

Artigo publicado em 30.03.2012. 